\title{
Influences of station length and inter-station distance on delays and delay propagation on single-track lines with regional rail traffic
}

\author{
O. Lindfeldt \\ Division of Traffic and Logistics, \\ Department of Transport and Economics, Royal Institute of Technology, \\ Stockholm, Sweden
}

\begin{abstract}
Train services on single-track lines suffer from time losses due to crossings, imposed by the bidirectional traffic. The time losses are caused by constraints in the infrastructure and delay propagation, which give a stochastic contribution that varies from one crossing situation to the other.

Two examples of infrastructure improvements that decrease the time loss are examined: increased track length at timetabled crossing stations and decreased inter-station distances. A mathematical model is used to evaluate these improvements.

Longer station tracks seem to be very efficient when traffic intensity and delay variances are moderate. Shortened inter-station distances give less effect but are less sensitive to delay variance and give valuable additional line capacity.

The model used assumes independence between crossing trains, which imposes a moderate capacity utilisation. In more congested situations simulation methods are needed to make more complex crossing patterns possible.
\end{abstract}

Keywords: single-track, delay propagation, partial twin-track, inter-station distance.

\section{Introduction}

On single-track lines train services often have lower average speed and worse punctuality than on twin-track lines. These facts are caused by the bidirectional traffic that calls for crossings. These crossings take place on crossing stations with two or more parallel tracks. In many cases the crossing itself leads to time 
losses, compared to a crossing on a twin-track line. These time losses consists of three parts:

- A static lowest time loss that is given by the infrastructure. This is a minimal (theoretical) time loss that occurs also when the crossing trains arrive at the station in an optimal way.

- A planned time loss that is due to infrastructure imperfections, such as unequal inter-station distances, irregular traffic or other types of planned time supplements.

- A stochastic time loss due to arrival delays, varying from one crossing situation to the other.

These three factors are not additive. High values on the planned time loss can overtake the static one and make the local infrastructure less constraining. A planned time loss also decreases the impact of arrival delays. One important case is when a crossing is combined with an ordinary stop for passenger exchange. In such situations influences of infrastructure constraints are often minimized and the impact of arrival delays decreased. Assuming perfect conditions in surrounding infrastructure, no time supplements and no stops for passenger exchange, the static lowest time loss and the stochastic time loss due to crossing can be analysed systematically.

Regional train services have some important demand features that are hard to combine with constraints imposed by single-track lines:

- Low travel times. Regional train services must reach a high average speed.

- High frequency. At least in morning- and afternoon rush hour a regular and frequent service is required.

- High punctuality. People travelling to and from their work places require a high punctuality.

Trying to meet these requirements, crossing restrictions is a great problem. This paper describes two examples of improvements that decrease time loss due to crossing: longer station tracks and shorter inter-station distances. Other measurements, such as higher speed in points etc, will be left to future research.

\section{Method}

\subsection{Model}

In order to analyse crossing phenomena on single-track lines, a mathematical model has been constructed. In the model it is possible to vary several parameters, hence different kinds of analyses can be performed. Most important variables are: inter-station distances, track lengths for each station, speed restrictions at points, interlocking time for each station, vehicle retardation and acceleration data and distributions of arrival delays (primary delays).

One advantage of this model is that one or more variables can be varied systematically. As shown later, characteristics of different infrastructure designs can be evaluated very easily. 
In order to make the model as simple and transparent as possible, some idealizing assumptions were made:

- Signalling system: ERTMS level 2, e.g. continuous update of driving permissions.

- Crossing trains are independent before crossing. This assumption requires a moderate capacity utilisation. At higher levels, interferences between trains will cause further restrictions on crossing. The model serves to give a first idea about characteristics of single-track lines. A very interesting analysis of highly congested cases will be performed by means of a simulation later on, in order to find limitations of this simple model.

- Everything that happens outside the model is taken into account by distributions of arrival delays. Each train only cross one other train in the modelled line.

- No time supplements in timetable. One output of the analysis is the need for (and effect) of time supplements.

- Dispatching follows the very simple rule to minimize the sum of added delay for crossing trains. This assumption also requires a moderate capacity utilisation.

- No gradients.

Given the infrastructure model, the vehicle model and the idealizing assumptions delay characteristics for a line can be calculated. Fig. 1 shows how the additional delay, due to crossing, depends on the difference in arrival delay.

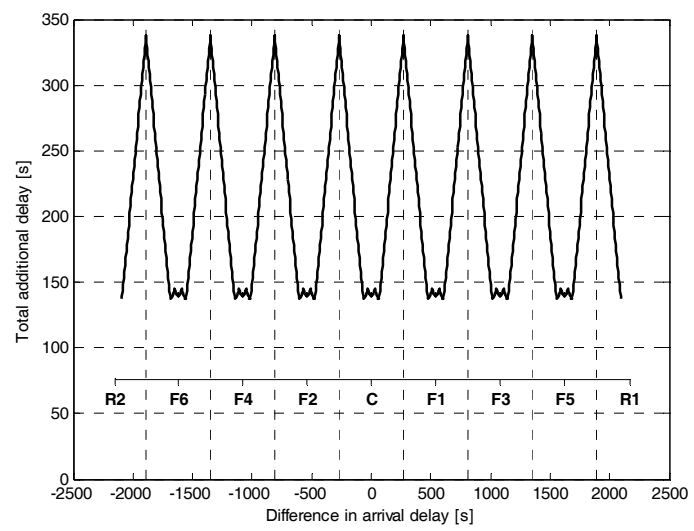

Figure 1: Total additional delay as a function of difference in arrival delay. Each crossing station is used during a time interval between two adjacent maxima (vertical dotted lines). Station signatures R2-R1 shown. $\mathrm{C}$ denotes timetabled crossing station.

For every five seconds of delay difference the model has solved a crossing conflict. This is done by first detecting the conflict and alternative crossing solutions. Second a crossing course, involving train movements, is performed for 
each station alternative. Finally the station giving lowest total additional delay is chosen to give the delay value for the current delay difference.

Note that the reference level, additional delay $=0$, represents a situation where both trains run the line without any time losses due to crossing, which corresponds to a twin-track line. In fig. 2 the tested line is schematically shown.

The length of the model, $120+20 \mathrm{~km}$, is chosen statistically so that at least $99 \%$ of all crossings will take place within the model, given that both distributions of arrival delays follow a specific worst-case distribution with very high variance, taken from the Swedish railway's delay data.

In solving crossing conflicts, the model has to choose the best station for each conflict. Therefore the border stations, B1 and B2 in fig. 2, must be treated carefully, since the model does not know anything about stations beyond these.

So far no attention has been paid to the fact that the difference in arrival delay follows a distribution. Thus the outcome of different differences of arrival delays will be given different weights according to a distribution. This distribution can be calculated through convolution of the two crossing trains' arrival delay distributions:

$$
f_{Z}(z)=\int f_{X}(\tau) f_{Y}(\tau-t) d \tau
$$

In eqn (1) $\tau$ denotes arrival delay for the down train, $t$ denotes arrival delay for up train and $z$ denotes difference in arrival delay. $f_{X}$ and $f_{Y}$ are the corresponding probability density functions and $f_{Z}(z)$ the resulting probability density function. Eqn (1) requires that the delays of two crossing trains are independent.

As a first step, negative exponential distributions have been used. Real delay statistics show that expected values on single-track lines in Sweden are 150-350 seconds. A negative exponential distribution, given the same expected value, shows a higher variance than empirical distributions. Later in the research project, other, more sophisticated distributions will be examined in order to get less discrepancy.

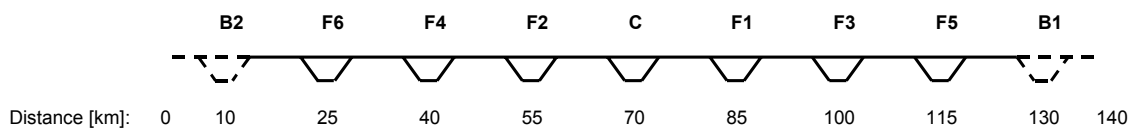

Figure 2: Example of line model with uniform inter-station distance $15 \mathrm{~km}$ and track length $750 \mathrm{~m}$ (Swedish standard).

Two equal negative exponential distributions $\left(f_{X}=f_{\mathrm{Y}}\right)$ give a Laplace distribution when convoluted, eqn (2). In fig. 3 this distribution is shown for three values on the scale parameter $\lambda$. Corresponding expected values for $f_{X}$ and $f_{\mathrm{Y}}$ were 150,250 and $350 \mathrm{~s}$ respectively. Note that the same distribution has been used for down- and up trains, making the curves symmetrical.

$$
f_{Z}(z)=\frac{\lambda}{2} e^{-\lambda|z|} .
$$




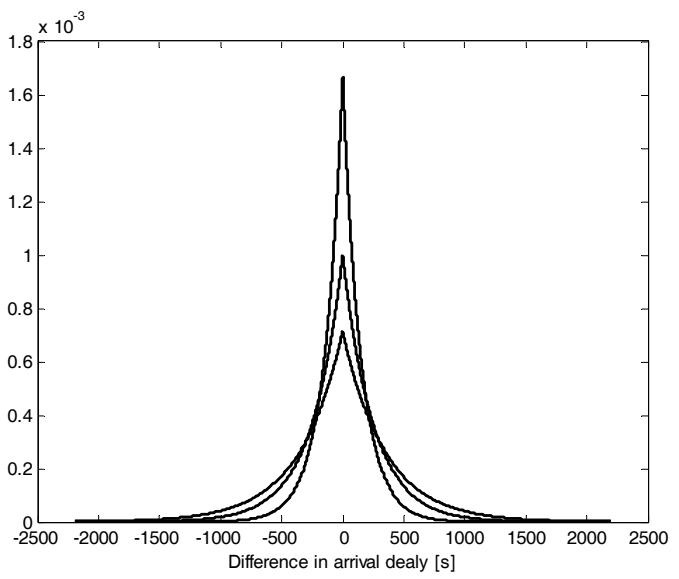

Figure 3: Three levels of probability density functions for difference in arrival delay. Standard deviation 210, 350 and $475 \mathrm{~s}$.

A very interesting question is how sensitive the outcome of different infrastructure improvements is for the shape of this distribution. Some measures will turn out to be quite dependent, while others will not.

\subsection{Choice of variables}

Several improvements are possible in order to get less time consuming crossings and lower disturbance sensitivity. Two interesting alternatives are:

- Increased track lengths at the station where the crossings are planned to take place. When station tracks are longer than a certain length, crossings without any interference between trains are possible. To make this kind of improvement cost efficient, some kind of regularity with a basic interval timetable is required so that utilisation is high. This timetable also has to be stable for several years. A severe drawback for these long stations is just that the stations on the line have different performance. Choosing another timetable, whose crossing pattern differs from the optimal one, results in low infrastructure utilisation.

- Decreased inter-station distances. If inter-station distances are decreased the maximum additional delay will be lower, but the minimum additional delay will not change. An important advantage of decreased inter-station distances is that it increases the overall capacity of the line. This means that the investment can be used either to run more trains, maintaining existing punctuality, or to run the same number of trains as before the improvement but with a higher punctuality.

To evaluate the effect of increasing track length at the timetabled crossing station, $\mathrm{C}$ in fig. 1 and 2, track length was increased stepwise by $500 \mathrm{~m}$ from $750 \mathrm{~m}$ to $28250 \mathrm{~m}$. Thus inter-station distances F2-C and C-F1 decreased by 250 $\mathrm{m}$ each time. For moderate increases $(\leq 15 \mathrm{~km})$ this method seems to be of 
practical interest. The shortened inter-station distances on each side of station $\mathrm{C}$ is then part of the total improvement. For longer extensions the question is whether to adjust flanking inter-station distances for capacity or economical reasons.

The other variable of special interest, inter-station distance, requires a different approach. In this case all stations, $\mathrm{C}$ included, were of same length: $750 \mathrm{~m}$. An interval of interesting inter-station distances was defined as $3-15 \mathrm{~km}$. Between these limits calculations has been performed with a step of $500 \mathrm{~m}$. As already mentioned, a minimum model length of $120+20 \mathrm{~km}$ was set up using delay statistics. Given this minimum, the number of modelled stations increased with decreasing inter-station distances. Moreover, the number of stations had to be odd (symmetry reasons) and so this theoretical number, 120/inter-station distance, must be rounded upwards to nearest odd integer.

These adjustments result in different total model length for different interstation distances, all of them $\geq 140 \mathrm{~km}$. The varying model length could make comparisons more difficult, but since the probability of crossing outside the middle- $120 \mathrm{~km}$ model part is negligible, this will not cause any real problems.

\section{Results}

\subsection{Increased track length of timetabled crossing station}

Fig. 4 shows how the total additional delay depends on the difference in arrival delay. The upper, solid, curve represents a reference case with $15 \mathrm{~km}$ interstation distances and $750 \mathrm{~m}$ track length for all stations (shown in fig. 2). Note that each station, located between two adjacent maxima, has a centred interval with low additional delay of about $140 \mathrm{~s}$. At these intervals both trains arrives at the station so simultaneously that none of them has to brake all way to stop. On the slopes up towards a maximum, where the crossing is moved to the adjacent station, one train has to stop and wait, which gives linearity. On the slopes stochastic time loss is dominating, whereas in the minimum areas infrastructure constraints, such as speed restrictions in points and signal interference, due to short station tracks, is the dominating cause of additional delay.

If track length increases, the curve for total additional delay will shift in the time interval covered by station $\mathrm{C}$. This is shown in fig. 4. Equidistance between two doted curves is $4 \mathrm{~km}$, so that the upper one represents a track length of $4750 \mathrm{~m}$ and the lower $24750 \mathrm{~m}$. Due to all symmetries in the model parameters the result is quite easy to understand:

- Maxima surrounding the extended station get lower. This is a result of shortened inter-station distances on each side. Note that doted curves coincide with the reference curve out to the left, and right, of intersection at the new maxima. This means that an extension only affects a specific (local) time interval.

- Curves corresponding to longer station tracks get a new, lower and wider minimum (90 s). On this minimum level time loss depends only on infrastructure constraints, e.g. speed restrictions at entrance and exit 
points for one of the crossing trains. Within a time interval crossing can take place without signal interference between the trains. The length of this interval is equal to the width of the minimum area, which depends on the track length.

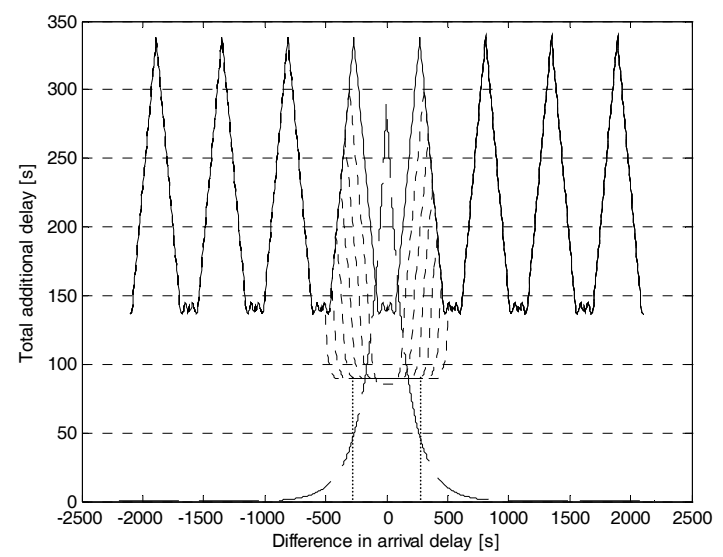

Figure 4: Total additional delay as a function of difference in arrival delay.

Fig. 4 also shows the probability density function for difference in arrival delay (standard deviation $210 \mathrm{~s}$ ), dashed line reaching 0 -level at $\pm 1000 \mathrm{~s}$. Note that a track length of $16750 \mathrm{~m}$ covers a big share of the area below the density curve (between vertical lines). The effect of an extension is thus double. First the minimum level becomes lower, leaving only static infrastructure factors. Second the interval within which the minimum value occurs becomes wider. A greater share of crossings gets the minimum additional delay. These facts result in a strong decrease of average additional delay for the first kilometres of extension.

\subsection{Decreased inter-station distances}

The result of different inter-station distances is shown in fig. 5. The upper, solid curve still represents a reference case where all inter-station distances are set to $15 \mathrm{~km}$ and track length to $750 \mathrm{~m}$. A reduction to $9 \mathrm{~km}$ inter-station distances gives a second curve, doted; with maximum additional delay about $225 \mathrm{~s}$. A further reduction to $3 \mathrm{~km}$ gives a curve that oscillates around $140 \mathrm{~s}$, hard to distinguish in the figure.

The effect of decreasing inter-station distances is lower maxima and an increasing share that lies at minimum levels (140 s in this case). In fact, the decrease in maxima is linear from $15 \mathrm{~km}$ to ca $5 \mathrm{~km}$. In this interval stochastic delay propagation plays an important role to build up additional delay, although the static time loss is important in situations where trains arrive simultaneously to a station. The stochastic delay propagation is proportional to inter-station distance. 
At shorter distance, 3-4 km, the static time loss due to infrastructure constraints dominates and covers stochastic effects. Thus stochastic effects cannot be seen in fig. 5 for inter-station distance $3 \mathrm{~km}$.

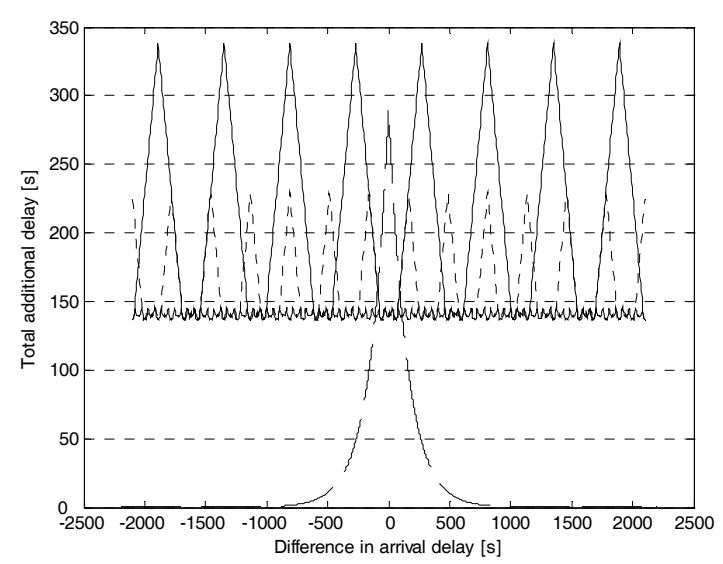

Figure 5: Total additional delay as a function of difference in arrival delay.

\subsection{Average total additional delay and standard deviation}

The average total additional delay is given by eqn 3 :

$$
\overline{T A D}=\int f_{Z}(z)^{*} g(z) d z .
$$

Here $z$ denotes difference in arrival delay, $f_{Z}$ is the probability density function and $g$ is the total additional delay function shown in fig. 4 and 5. Curves for average total additional delay are shown in fig. 6 . The results of three different $\mathrm{f}_{\mathrm{Z}}$-functions, standard deviation 210,350 and $475 \mathrm{~s}$, are shown.

In fig. 6 solid curves represent average total additional delay and dashed curves standard deviations for total additional delay. In the left part, showing results for increased station length, average of total additional delay falls from a maximal level of about $200 \mathrm{~s}$, consisting of static time loss and a great part stochastic delay propagation, towards a lowest static time loss (here $90 \mathrm{~s}$ ) where delay propagation is almost negligible. Note the effect of higher variance in difference of arrival delay that makes these curves start at a higher level and fall slower.

For the inter-station distance case the minimal total additional delay depends on the fact that these "short" stations always give time losses due to signal interference between crossing trains. Therefore these curves (right part of fig. 6) fall from maximal level towards this static lower limit determined by infrastructure performance. Note that a change in variance for the difference in arrival delay does not influence the average delay very much. 

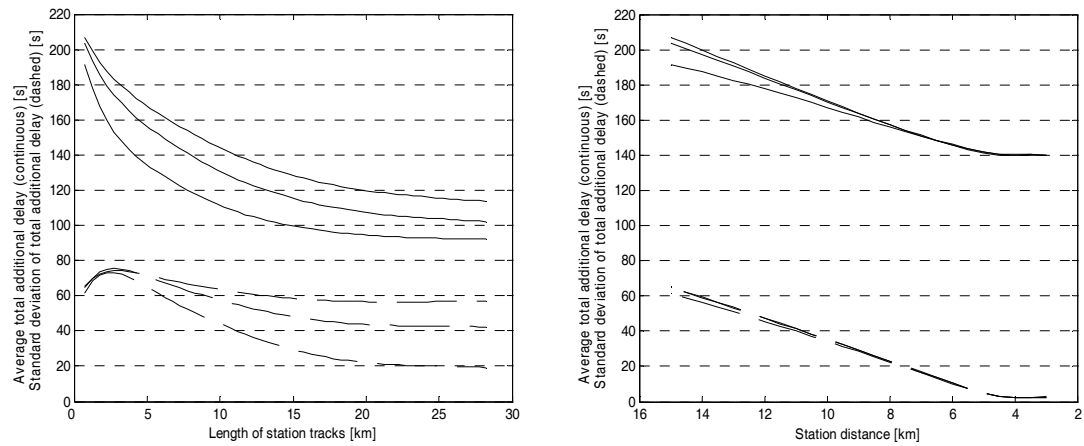

Figure 6: Average total additional delay (solid) and standard deviations of total additional delay (dashed) for increasing track length, left part, and decreasing inter-station distances, right part. In each case results from three different distributions (fig. 3) for difference in arrival delay are shown.

\section{Conclusions}

Presented results show some principle differences between the two alternative improvements.

Extended track lengths are most efficient when variance in arrival delay difference is low. A lower standard deviation of arrival delay difference than $210 \mathrm{~s}$, lowest curves in fig. 6, and thereby a higher precision, would make this improvement even more efficient. Still higher effect could be obtained with higher speeds in entrance and exit points at the extended station, resulting in lower minimum time loss. Shortened inter-station distances, especially values under $10 \mathrm{~km}$, seem to give a result that is more independent of variance of arrival delay difference. Both these results provide a moderate capacity utilisation, so that alternative crossing stations are available in most frequent crossing situations.

Extended track lengths only have a local effect. Rather high minimum levels of standard deviations reflect this. Crossing situations that fall outside the extended station still result in high additional delay due to long inter-station distances. In case of shorter inter-station distances standard deviations fall faster and to a much lower minimum level.

Low variance in arrival delay difference is an important requirement to obtain independence between crossing trains. Therefore it is very interesting to find out at which variance level, and/or capacity utilisation level, this simple model starts to give serious under-estimations of additional delays. This check will be done by simulation in RailSys, which makes interactions between several crossings possible through active dispatching. A qualified guess is that the simple model works quite well if traffic intensity is lower than three trains an hour and direction, provided that variance in arrival delay is moderate. 
The studied cases are quite idealized in themselves. Therefore further work will be done to examine asymmetric cases: between traffic directions, in the infrastructure etc. Extended stations are constructed for one (or a few) timetable structure(s). This is a very important (negative) property that can be examined by systematic change of planned crossing position.

A very important situation is when a (planned) crossing is combined with a regular stop for passenger exchange, which gives lower time loss. An analysis of such cases can point out how timetables should be constructed.

Fortunately this work will contribute to a deeper understanding of the features of single-track railway lines and the possibilities to get fast services with high punctuality on such lines. The complete work will be presented in a licentiate thesis in 2007.

\section{Acknowledgements}

This project has been financially supported by the Swedish National Rail Administration.

\section{References}

[1] Barter, W., Forecasting robustness of timetables, Comprail 2004.

[2] Fröidh, O., Introduction of regional high speed trains. A study of effects of the Svealand line on the travel market, travel behaviour and accessibility, 2003.

[3] Mattsson, L-G., Ways of deriving train delay relationships, proceeding from the $1^{\text {st }}$ International seminar on railway operations modelling and analysis, Delft, 2005.

[4] Wendler, E., On waiting times of scheduled train paths in non-cyclic timetables, proceeding from the $1^{\text {st }}$ International seminar on railway operations modelling and analysis, Delft, 2005. 